\title{
Analysis of Multimedia Traffic Performance in a Multi-Class traffic environment for UDP traffic class.
}

\author{
Dr. Nipun Sharma ${ }^{1}$, Er. Harshit ${ }^{2}$, Kiran Deep Singh ${ }^{3}$ \\ ${ }^{1}$ AP, Quest Group of Institutions, Mohali. \\ ${ }^{2} H O D$ and AP, ECE Department, DIET, Kharar, Mohali \\ ${ }^{3}$ Research Scholar, DIET, Kharar, Mohali.
}

\begin{abstract}
The computer networks have evolved themselves into an altogether new generation with Mobile AdHoc networks. The Mobile Ad-Hoc Networks are increasingly becoming more sophisticated and complex in terms of topology, routing and security[1][2][3]The new age MANETs incorporate routing of heavier traffic classes like audio, video and multimedia. It has become important to study the performance characteristics of the multimedia traffic class in MANETS [10] that includes packet loss rate and throughput. In this paper we will discuss these performance parameter throughput under different scenarios like varying Bandwidth, channel error rate, delay and fragment size.
\end{abstract}

Keywords: MANET, Packet loss rate, Throughput, Bandwidth, Fragment size

\section{Introduction}

In this paper we present a relatively complex network with both UDP and TCP traffic flowing in the network. If we study the performance of UDP traffic class in a network, it becomes easier to predict the behaviour of multimedia traffic class as it will be sent over the UDP in the simulations[9]

We analyse the UDP traffic on the main router to router link for the following parameters.

- Throughput: Throughput or network throughput is number of bits delivered successfully per second to the destination. It is the sum of bits received successfully by all destinations. This data may be delivered over a physical or logical link. The throughput is measured in bits per second (bps).

Mathematically, it can be defined as:

\section{Throughput $=$ N/1000}

Where $N$ is the number of bits which is received successfully by all destinations [4]

\section{Methodology}

The primary traffic class that multimedia adopts is UDP. IN multimedia communication for MPEG-4 traffic, if we analyse the characteristics of UDP traffic for throughput and other network performance parameters then we can predict the behaviour of MPEG-4 also in a network with multi-class traffic. For this purpose a n/w generating both TCP and UDP traffic classes is created and tested.

The topology of the network created is as follows:

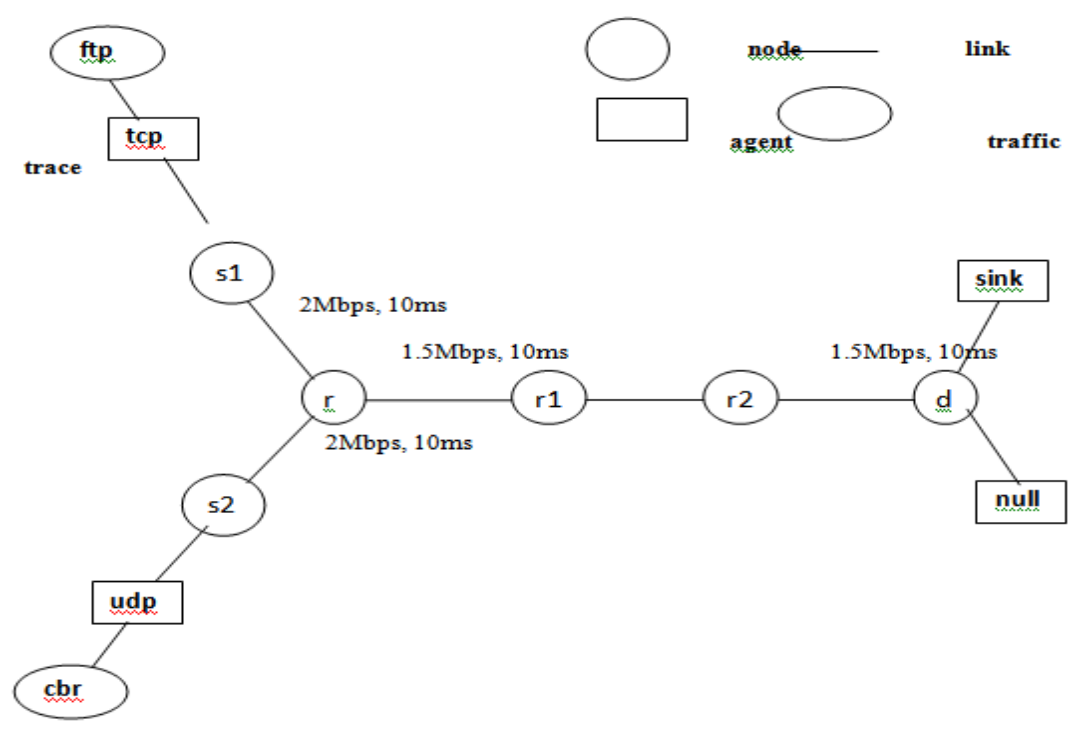


The network has 2 source nodes (S1 and S2), one generating TCP traffic and the other generating UDP traffic to be transferred at the destination node (d) [7][8] The network parameters are changed on the link (r1-r2) as shown in the figure.

The following parameters are changed in the network. In first case, the bandwidth of the main router to router link (r1-r2) is changed keeping the other network parameters unchanged. In the second scenario the delay of the main router to router link (r1-r2) is changed keeping the other network parameters unchanged. In the third scenario the error rate of the main link is changed and in the fourth scenario the packet size is changed.

The result that we present is the effect of fragment size on THROUGHPUT. We have kept the delay to be constant and analysed with varying bandwidths and error rate.

We observed the throughput for different fragment sizes at $10 \mathrm{~ms}, 50 \mathrm{~ms}$ and $100 \mathrm{~ms}$ delay

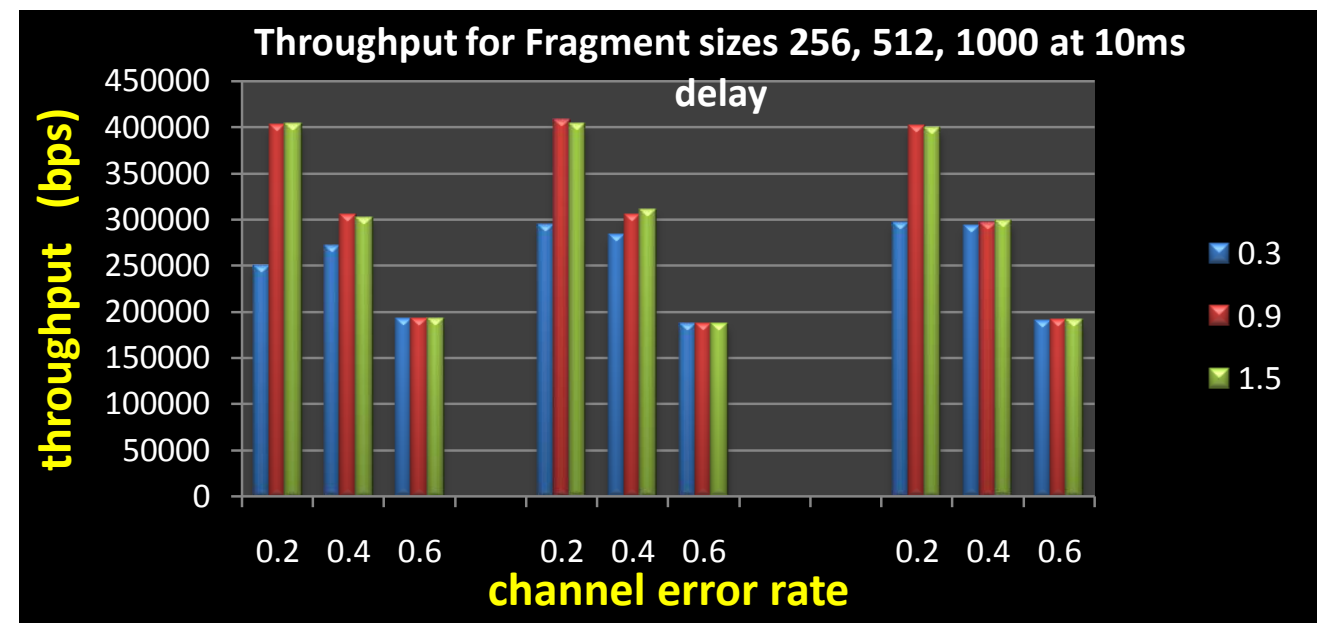

Fig 1:Analysis of Throughput at various fragment sizes for varying delays and Bandwidths for $10 \mathrm{~ms}$ Delay

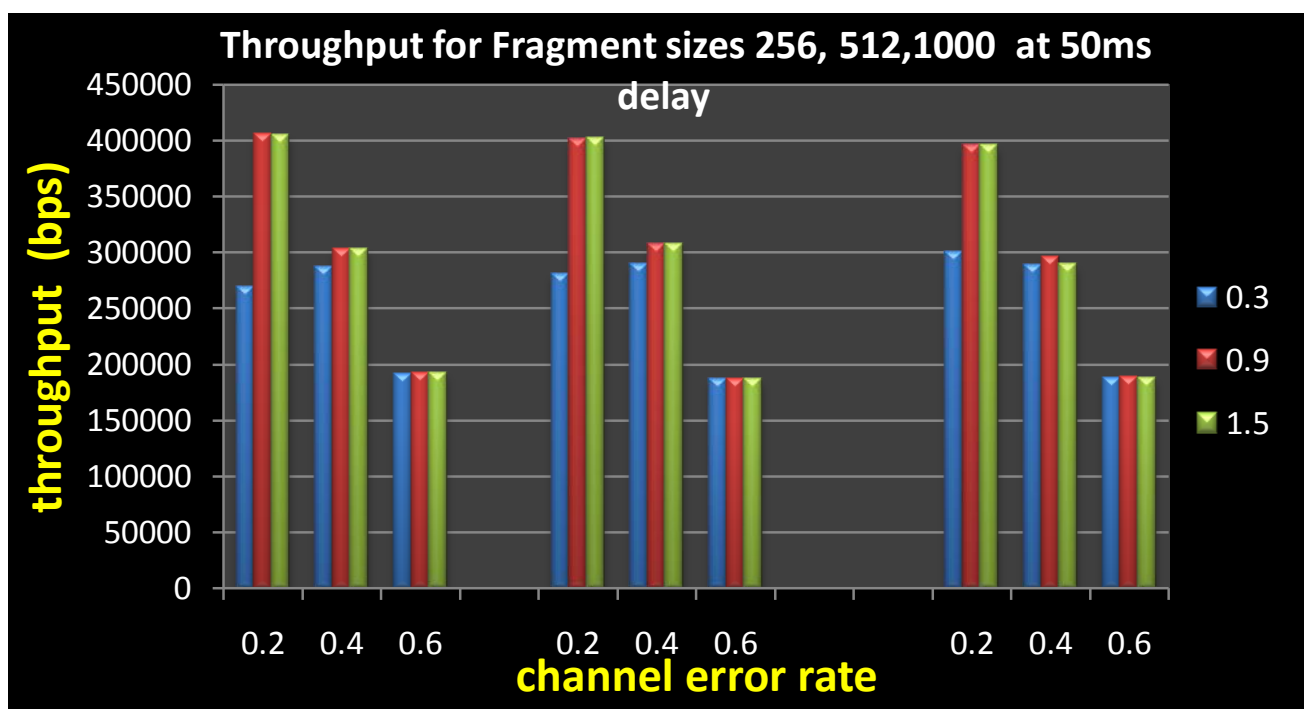

Fig 2:Analysis of Throughput at various fragment sizes for varying delays and Bandwidths for $\mathbf{5 0}$ ms Delay 


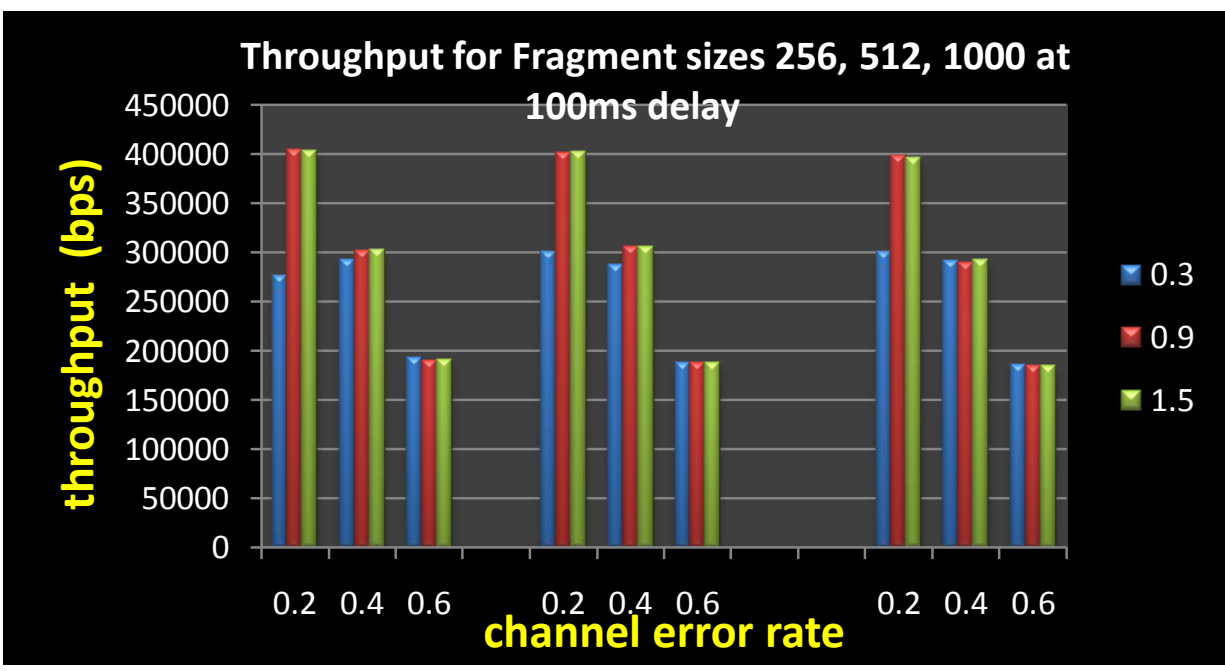

Fig 3:Analysis of Throughput at various fragment sizes for varying delays and Bandwidths for $100 \mathrm{~ms}$ Delay

\section{Conclusion and Future scope}

In this paper we find out the effect of different fragment sizes having varying bandwidth, delays and channel error rate on the throughput based on above topology. The results reveal that the throughput increases as the fragment size increases at lower error rates and throughput decreases when the fragment size increases at higher error rates. The results may facilitate careful optimization of multimedia traffic over UDP. The behaviour of UDP traffic largely depend upon the fragment size. The effect of fragment size on throughput for varying bandwidth, delay and channel error rate provides a complete estimation of traffic control.

\section{References}

[1] S. P. Alampalayam, A. Kumar, and S. Srinivasan, "Mobile ad hoc networks Security - a taxonomy," in Proceedings of ICACT Conference, 2005, pp. 839-844.

[2] Naruephiphat, W, "Routing Algorithm for Balancing Network Lifetime and Reliable Packet Delivery in Mobile Ad hoc Networks," in ubiquitous, Autonomic and Trusted Computing, conference publications, 2009

[3] Nipun Sharma, "ANALYSIS OF SECURITY REQUIREMENTS IN WIRELESS NETWORKS AND MOBILE AD-HOC NETWORKS" in GESJ: Computer Science and Telecommunications 2010| Vol No.5 (28)

[4] Pankaj Rohal, Ruchika Dahiya, Prashant Dahiya, “ Study and Analysis of Throughput, Delay and Packet Delivery Ratio in MANET for Topology Based Routing Protocols (AODV, DSR and DSDV)” in international journal for advance research in engineering and technology Vol. 1, Issue II, Mar. 2013 ISSN 2320-6802

[5] http://en.wikipedia.org/wiki/Packet_loss

[6] Yi Lu Yuhui Zhong, "Packet Loss in Mobile AdHoc Networks"Bharat Bhargava Purdue University, bb@cs.purdue.edu

[7] http://csie.nqu.edu.tw/smallko/ns2/measure.htm

[8] Network Simulator 2 - http://www.isi.edu/nsnam/ns/

[9] Dr. Nipun Sharma,Ms. Jasdeep Kaur , Ms. Jasmine Yadav, “ Implementation and Analysis of Fragmentation of MPEG-4 Traffic in NS-2 integrated with Evalvid" Oeconomics of Knowledge, Volume 5, Issue 2, Spring 2013.

[10] Vasos Vassiliou, Pavlos Antoniou, Iraklis Giannakos, and Andreas Pitsillides, "Requirements for the Transmission of Streaming Video in Mobile Wireless Networks" 